\title{
Yolk carotenoids increase fledging success in great tit nestlings
}

\author{
Viviana Marri $\cdot$ Heinz Richner
}

Received: 26 February 2014 / Accepted: 13 August 2014 / Published online: 21 August 2014

(C) Springer-Verlag Berlin Heidelberg 2014

\begin{abstract}
Avian mothers can influence offspring phenotype through the deposition of different compounds into eggs, such as antibodies, hormones and antioxidants. The concentration of carotenoids in yolk is larger than in maternal plasma, suggesting an important role of these compounds for offspring development. Since carotenoids have to be acquired from the diet, they may be available in limiting amounts to the mothers. Here, we investigated the role of egg carotenoids for offspring growth by experimentally increasing the concentration of yolk lutein, the main carotenoid in great tit (Parus major) yolk. We subsequently measured body condition, oxidative stress, immune response, plumage colouration and fledging success. Lutein increased body mass soon after hatching and fledging success, but did not affect tarsus length, oxidative stress, immune response and plumage colouration. The higher content of yolk lutein could have increased body mass by reducing oxidative stress caused by high metabolic rates of rapidly growing embryos or by promoting cell differentiation and proliferation. The positive effect of lutein on fledging success seems to be mediated by its influence on body mass 3 days post-hatch, since these two traits were correlated. The finding that our treatment did not affect traits measured later in the nestling period, except for fledging success, suggests that yolk lutein has short-term effects that are essential to increase survival until fledging. Our study shows the positive effect of yolk lutein on offspring survival in the great
\end{abstract}

Communicated by Markku Orell.

V. Marri $(\bowtie) \cdot H$. Richner

Evolutionary Ecology Lab, Institute of Ecology and Evolution, University of Bern, Baltzerstrasse 6, 3012 Bern, Switzerland

e-mail: viviana.marri@iee.unibe.ch tit, and therefore suggests an important role of carotenoidmediated maternal effects.

Keywords Egg $\cdot$ Lutein $\cdot$ Maternal effects $\cdot$ Offspring survival · Parus major

\section{Introduction}

Maternal effects occur when maternal phenotype or the environment experienced by the mother influence the offspring phenotype (Mousseau and Fox 1998). Females can change prenatal environment of the nestlings through the deposition of different compounds such as antioxidants (Surai 2002), antibodies (Hasselquist and Nilsson 2009) and hormones (Groothuis et al. 2005) into eggs. In birds, the concentration of carotenoids in yolk is larger than in maternal plasma (Saino et al. 2002), suggesting an important role of these compounds for embryos and hatchlings. Carotenoids are pigments synthesized by plants that animals have to acquire from food (Goodwin 1984), and hence they may be available in limiting quantities. In many studies supplementing laying mothers with carotenoids resulted in higher yolk carotenoid concentration (e.g. McGraw et al. 2005; Ewen et al. 2009; Berthouly et al. 2008) and higher phenotypic quality of the offspring (e.g. Biard et al. 2005; Koutsos et al. 2006; Berthouly et al. 2008). These studies therefore suggest that carotenoid concentration in the yolk may be limiting.

Carotenoids have different physiological functions: they are involved in cell differentiation and cell proliferation (reviewed in Surai 2002), and they can regulate and stimulate immune functions (Møller et al. 2000). Moreover, carotenoids have been claimed to reduce oxidative stress, defined as the imbalance between reactive oxygen species 
(ROS) and antioxidants in favour of the former (Sies 1991). However, the antioxidant role of carotenoids has been shown in vitro, but their importance in vivo was recently questioned (Costantini and Møller 2008). Carotenoids are also responsible for the typical yellow to red colouration of many signals used in sexual selection (Hill 1991), and in parent-offspring communication (Saino et al. 2000).

Several experiments (e.g. Berthouly et al. 2008; Biard et al. 2005; Ewen et al. 2009; McGraw et al. 2005) have studied the effect of yolk carotenoids on offspring by manipulating carotenoid availability for females before egg laying. However, carotenoid-supplied females may also change the amount of other compounds allocated to the eggs (Surai et al. 2003), and could invest carotenoids differentially according to sex or laying order. Additionally, carotenoids could influence the resolution of maternal trade-offs (Bertrand et al. 2006). Hence, such an indirect approach where females are supplemented will not allow disentangling these effects from the direct effects mediated by yolk carotenoids, making the interpretation of the findings more complicated.

With this study we aimed at understanding the importance of yolk carotenoids for offspring growth and development in the great tit Parus major, a small-hole nesting passerine. We therefore injected lutein, the main carotenoid in great tit eggs (Remeš et al. 2011), and subsequently measured the expression of traits for which carotenoids are supposed to be important. To our knowledge only a few experiments directly manipulated carotenoid content in eggs (Romano et al. 2008; Saino et al. 2003; Saino et al. 2011), but none of them assessed simultaneously the effects on body condition, oxidative stress, immune status and feather colour. Since carotenoid availability for laying females seems to be limited, and since carotenoids are supposed to be involved in important biological functions, we expect that a direct increase of lutein content in eggs would increase the viability of nestlings after hatching.

\section{Materials and methods}

This experiment was performed during spring 2012 in a natural population of great tits, breeding in nest boxes in Könizbergwald, a forest near Bern, Switzerland $\left(46^{\circ} 56^{\prime} \mathrm{N}\right.$, $7^{\circ} 24^{\prime} \mathrm{E}$ ). Nest-boxes were visited regularly from the beginning of the breeding season to determine the start of egg laying.

\section{Lutein treatment}

Whole broods were randomly assigned to two treatments: lutein or control group. After the fifth egg in the laying sequence, we injected all the eggs with either $2 \mu \mathrm{g}$ lutein
(floraGLO lutein $20 \%$ liquid in safflower oil) diluted in $2.5 \mu 1$ of safflower oil or with $2.5 \mu 1$ of safflower oil as a control. Every additional egg laid over the following days was treated in the same way. All eggs were therefore manipulated before incubation or at the very beginning of the incubation period. The injected dose of lutein corresponds to about $1 \mathrm{SD}$ of the average amount of lutein per yolk (mean $\pm \mathrm{SD}=3.86 \pm 1.84 \mu \mathrm{g}$ per yolk) found in a sample of eggs $(n=109)$ from the same population (Helfenstein, unpublished data). In the field, eggs were placed on a cold light source to identify the position of the yolk and, after disinfecting a small spot on the eggshell, we used a $25-\mu 1$ syringe (Hamilton 702LT) with a $25-G$ needle to inject the oil, either alone or mixed with lutein, into the yolk. After the injection we sealed the hole with a small drop of tissue adhesive (GLUture Topical Tissue Adhesive; Abbott Laboratories, North Chicago, IL).

We injected a total of 647 eggs, from which 331 nestlings hatched. The overall hatching success was $51.2 \%$ and it did not differ between treatments $\left(\chi_{1}^{2}=1.1, P=0.29\right.$, $n=91$ ). To assess the invasiveness of our egg injection we compared hatching success of our injected eggs with natural hatching success of a non-manipulated control. The natural hatching success of non-injected clutches was $88.4 \%$, therefore the injection decreased the hatching success $\left(\chi_{1}^{2}=86, P<0.0001, n=127\right)$, as observed in previous studies using a similar protocol (e.g. Ruuskanen et al. 2012; Podlas et al. 2013).

\section{Morphological measurement}

On day 3 post-hatch we measured nestling body mass with an electronic balance $( \pm 0.1 \mathrm{~g})$, and took a drop of blood from the metatarsus vein, which was then stored in ethanol $96 \%$ until later analyses to determine sex [see Griffiths et al. (1998) for the sexing technique]. Eight and 14 days post-hatch, we measured body mass $( \pm 0.1 \mathrm{~g})$ and tarsus length $( \pm 0.1 \mathrm{~mm})$.

Oxidative stress analyses

On day 12 post-hatch a blood sample $(20 \mu l)$ from the brachial vein was taken, kept cool in a ice box until centrifugation in the evening, and then stored at $-20^{\circ} \mathrm{C}$. We assessed oxidative damage using the Diacron reactive oxygen metabolite test (dROM test; Diacron International, Grosseto, Italy), which measures plasmatic ROM. ROM are the primary products of the oxidative cascade, caused by the exposure of biomolecules, especially lipids, to ROS. ROM have been used as a marker of early oxidative damage in several studies (e.g. Costantini 2006; Saino et al. 2011), moreover they are themselves pro-oxidants and can propagate the oxidation cascade (Halliwell and Gutteridge 2007). 
The plasma $(5 \mu \mathrm{l})$ was diluted with $200 \mu \mathrm{l}$ of a solution containing $0.01 \mathrm{M}$ acetic acid/sodium acetate buffer $(\mathrm{pH}$ 4.8 ) and a $N, N$-diethyl-p-phenylenediamine as a chromogen. The solution was then incubated for $75 \mathrm{~min}$ at $37{ }^{\circ} \mathrm{C}$. During the incubation the acidic $\mathrm{pH}$ favoured the release of $\mathrm{Fe}$ and $\mathrm{Cu}$ from plasma proteins that catalysed the cleavage of hydroperoxides into two different free radicals. These radicals reacted with the chromogen, producing a pink colour, whose intensity was directly proportional to the concentration of ROMs. The absorbance was read with a spectrophotometer at $490 \mathrm{~nm}$. The concentration of ROMs was calculated by comparison with a standard curve obtained by measuring the absorbance of a standard sample and a blank (distilled water). Measurements were expressed as millimoles per litre of $\mathrm{H}_{2} \mathrm{O}_{2}$ equivalents. Since available plasma quantity was limited, we run on each plate duplicates of a sub-sample of 17 nestlings only. The intra-assay repeatability sensu Lessels and Boag (1987) was high and significant $(r=0.98, P<0.0001)$.

Total antioxidant capacity of plasma was measured using the OXY-adsorbent test (Diacron International). This kit uses a colorimetric determination to quantify the ability of the plasma antioxidant barrier to cope with the oxidant action of hypochlorous acid. The plasma was diluted 1:100 with distilled water, $2 \mu 1$ of this solution was incubated with $200 \mu \mathrm{l}$ of a titred $\mathrm{HClO}$ solution at $37{ }^{\circ} \mathrm{C}$ for $10 \mathrm{~min}$. Then $5 \mu \mathrm{l}$ of chromogen, an alkyl-substituted aromatic amine solubilised in a chromogenic mixture, was added. This amine was oxidized by the residual $\mathrm{HClO}$ and transformed into a pink-colour derivative. The intensity of the coloured complex, which was inversely related to the antioxidant capacity, was measured by a spectrophotometer at $490 \mathrm{~nm}$. A blank sample (5 $\mu 1$ of distilled water) and a standard sample were used as reference; measurements were expressed as millimoles of $\mathrm{HClO}$ neutralized. All the samples were run in duplicate, the intra-assay repeatability, sensu Lessels and Boag (1987), was high and significant $(r=0.79, P<0.0001)$.

\section{Immune response}

On day 14 post-hatch we injected subcutaneously $0.01 \mathrm{mg}$ of lipopolysaccharide (LPS; from the cell membrane of Escherichia coli) dissolved in $0.02 \mathrm{ml}$ of phosphate-buffered saline in the left wing web. We measured the wing web thickness before and $24 \mathrm{~h}$ after the injection at the inoculation site with a constant-tension dial micrometer to the nearest $0.01 \mathrm{~mm}$. The wing web thickness was measured three times and the mean was used. The strength of the immune response was calculated as the difference between the value before and after the injection; a greater swelling reflects a better immune response (Parmentier et al. 1998). LPS induces an inflammatory response without producing the negative effects of a pathogen, allowing us to evaluate the cost of immune activation alone (Bonneaud et al. 2003).

\section{Breast colour}

On day 14 post-hatch, we collected from every nestling six feathers from each of two patches, located on both side of the chest, and stored them in small plastic bags kept in the dark. Later in the lab, we placed the feathers on top of each other on a black velvet surface before proceeding with spectrophotometric measurements. Spectral measures were made using a spectrophotometer (USB 4000; Ocean Optics), a bifurcated reflectance probe with a $200-\mu \mathrm{m}$ fiber core diameter (FCR-7UV200-2-ME) and a balanced deuterium tungsten-halogen light source (DH-2000-BAL; Ocean Optics). The probe was fitted with a black cylinder to standardize the measuring distance and exclude ambient light, and it was held perpendicular to the surface of the feathers. We recorded three reflectance spectra (each of these is the average of four scans with 100-ms integration time) per patch using Spectrasuite software version 1.0 (Ocean Optics). We averaged the measurements per repetition and then per patch to describe each individual (intrapatch repeatability, $r=0.89, P<0.0001)$. We calibrated the spectrophotometer with a diffuse reflectance standard (WS-1; Ocean Optics) before each patch was measured.

Colour vision in birds depends on four types of single cones: UV sensitive, short-wave sensitive (SWS), medium-wave sensitive, long-wave sensitive. Using Hadfield's SPEC package (2005), we estimated the amount of light captured by each of these cones, taking into account the sensitivity of the retinal cones, the transmittance properties of the ocular media and ambient light. We used the cone spectral sensitivities and ocular media transmittance reported for the blue tit Cyanistes caeruleus (Hart et al. 2000), the daylight irradiance spectrum, and we applied the von Kries algorithm to account for colour constancy. We then calculated the SWS ratio (Evans et al. 2010), an index of chromatic reflectance based on opponent processing that compares the quantum catch of the SWS single cones with the mean of the other three. The SWS ratio estimates the size of the trough in the violet-blue region of the reflectance spectra, caused by the selective absorption of carotenoids. The SWS ratio therefore increases with higher carotenoid content of feathers and is highly positively correlated with carotenoid chroma $(r=0.84, P<0.0001)$, a known measure of the amount of pigment deposited in the feathers (Saks et al. 2003).

\section{Statistical analyses}

The effects of lutein injection on nestling body mass, tarsus length, antioxidant capacity, oxidative damage, immune 
response and chromatic reflectance, were analysed using linear mixed-effect models with restricted maximum-likelihood estimation. To normalize data, we log transformed SWS ratio values and square root transformed the concentration of ROM. In all the models we included nest identity as a random factor to control for the non-independence of nestlings growing in the same nest. We also included sex, treatment and the interaction between sex and treatment as fixed factors to test for a possible sex-specific effect. Moreover, in all the models we included as covariates hatching date and brood size to test for possible seasonal and sibling competition effects. Finally, we included mass at day 14 when analysing colouration and immune response, as these traits can be condition dependent.

Fledging success, calculated as the proportion of hatchlings that fledged, was analysed using a generalized linear model with a quasi-binomial distribution to account for overdispersion. The model included treatment, brood size and hatching date. Additionally, we analysed fledging probability of individual nestlings using a generalized linear mixed model with a binomial error structure. The model included treatment and sex as fixed factors, brood size and hatching date as covariates and nest identity as a random factor

Models were simplified following a backward stepwise elimination procedure. Non-significant parameters were backward eliminated, starting with the interactions and then going on with the least significant variables. In the Results section $P$-values of non-significant terms are those just before removal from the model and $P$-values of significant terms are those from the reduced models. All the analyses were performed with $\mathrm{R}$ version 2.15.1 ( $\mathrm{R}$ Development Core Team 2010). We used the function lme from the nlme package for linear mixed effect models, the function glm for generalised linear model and the function lmer from the lme4 package for generalised linear mixed models.

\section{Results}

Body mass and size

Three days after hatching lutein-treated nestlings were significantly heavier $\left(F_{1,208}=4.4, P=0.04\right)$ than control ones (Fig. 1). Sex, hatching date, brood size or the interaction between treatment and sex did not significantly predict mass at day 3 (all $P>0.2$ ). Mass and tarsus 8 and 14 days post-hatching were not affected by lutein treatment or by the interaction between treatment and sex (Tables 1,2). Mass was negatively correlated with brood size both at days 8 and 14 (Table 1), while tarsus was correlated with brood size only on day 14 (Table 2). Males at day 14 were heavier (Table 1) and had longer tarsi (Table 2) than females.

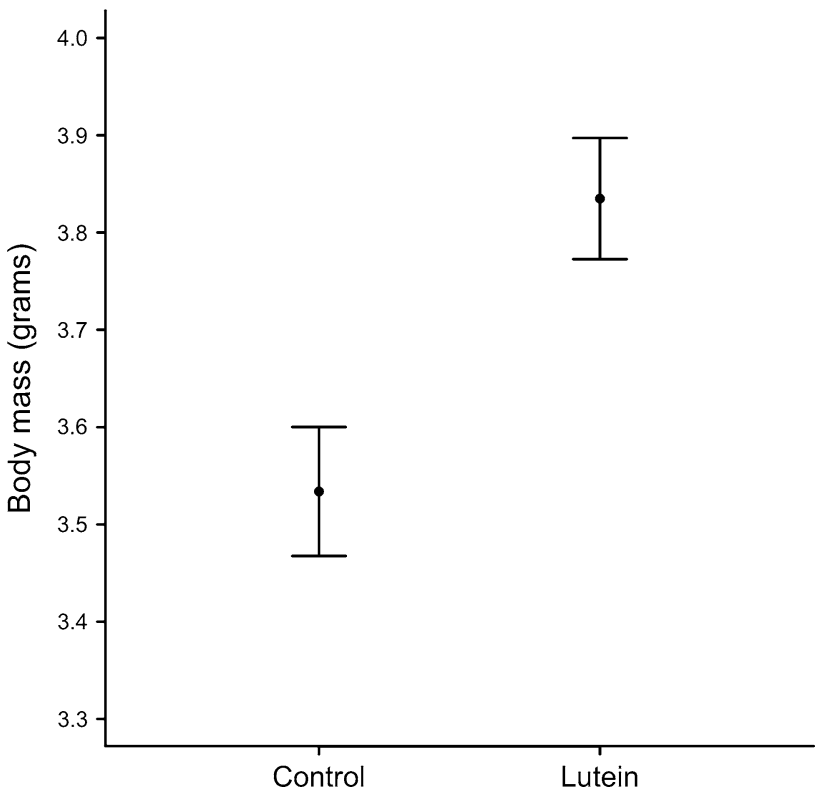

Fig. 1 Body mass (mean \pm SE; g), on day 3 post-hatch in relation to the treatment

\section{Oxidative stress}

The interaction between treatment and sex, and treatment alone, did not affect antioxidant capacity and oxidative damage (all $P>0.27$ ). Both antioxidant capacity and oxidative damage increased with hatching date $\left(F_{1,54}=7.6\right.$, $P=0.008$, estimate $\pm \mathrm{SE}=2.42 \pm 0.87 ; F_{1,54}=5.7$, $P=0.02$, estimate $\pm \mathrm{SE}=0.03 \pm 0.01)$, but did not show any correlation with sex or brood size (all $P>0.23$ ).

Immune response

We did not find a significant effect of the interaction between treatment and sex, and of lutein treatment on the swelling response to LPS injection $\left(F_{1,154}=01.8, P=0.18\right.$, $\left.F_{1,61}=0.84, P=0.36\right)$. The swelling response tended to be higher in heavier nestlings $\left(F_{1,156}=3.7, P=0.056\right.$, estimate $=0.75 \pm 0.39$ ), showed a weak non-significant correlation with hatching date $\left(F_{1,60}=3, P=0.088\right)$ and was unrelated to $\operatorname{sex}\left(F_{1,155}=0.31, P=0.57\right)$.

Breast plumage colour

Chromatic reflectance of breast plumage was not affected by lutein treatment $\left(F_{1,60}=0.03, P=0.86\right)$ or by the interaction between treatment and $\operatorname{sex}\left(F_{1,165}=0.39\right.$, $P=0.53$ ), but positively correlated with hatching date $\left(F_{1,60}=31.3, P<0.0001\right.$, estimate $\left.\pm \mathrm{SE}=0.02 \pm 0.004\right)$ and negatively correlated with brood size $\left(F_{1,60}=5.6\right.$, $P=0.02$, estimate $\pm \mathrm{SE}=-0.04 \pm 0.016$ ). Males showed 
Table 1 Linear mixed-effect model testing the effect of lutein treatment on body mass (g) 8 and 14 days after hatching

\begin{tabular}{|c|c|c|c|c|c|c|}
\hline \multirow[t]{2}{*}{ Effect } & \multicolumn{3}{|l|}{ Body mass day 8} & \multicolumn{3}{|c|}{ Body mass day 14} \\
\hline & Estimate $\pm \mathrm{SE}$ & $F_{\mathrm{df}}$ & $P$ & Estimate $\pm \mathrm{SE}$ & $F_{\mathrm{df}}$ & $P$ \\
\hline Intercept & $12.99 \pm 0.54$ & - & - & $17.36 \pm 0.49$ & - & - \\
\hline Treatment & $0.22 \pm 0.36$ & $0.37_{1,70}$ & 0.54 & $0.17 \pm 0.32$ & $0.3_{1,61}$ & 0.59 \\
\hline $\operatorname{Sex}$ & $0.22 \pm 0.15$ & $2.27_{1,198}$ & 0.13 & $0.52 \pm 0.16$ & $10.5_{1,168}$ & 0.0014 \\
\hline Hatching date & $-0.02 \pm 0.03$ & $0.48_{1,69}$ & 0.49 & $-0.008 \pm 0.02$ & $0.09_{1,60}$ & 0.77 \\
\hline Brood size & $-0.4 \pm 0.11$ & $14.2_{1,70}$ & 0.0003 & $-0.40 \pm 0.1$ & $17.4_{1,61}$ & 0.0001 \\
\hline Treatment $\times$ Sex & $-0.17 \pm 0.3$ & $0.33_{1,197}$ & 0.56 & $0.17 \pm 0.33$ & $0.26_{1,167}$ & 0.61 \\
\hline
\end{tabular}

$F$ - and $P$-values of non-significant terms are those just before removal from the model. Terms retained in the final model are highlighted in italic. Reference level for coefficients is a female under control treatment

Table 2 Linear mixed-effect model testing the effect of lutein treatment on tarsus length $(\mathrm{mm}) 8$ and 14 days after hatching

\begin{tabular}{|c|c|c|c|c|c|c|}
\hline \multirow[t]{2}{*}{ Effect } & \multicolumn{3}{|c|}{ Tarsus length day 8} & \multicolumn{3}{|c|}{ Tarsus length day 14} \\
\hline & Estimate $\pm \mathrm{SE}$ & $F_{\mathrm{df}}$ & $P$ & Estimate $\pm \mathrm{SE}$ & $F_{\mathrm{df}}$ & $P$ \\
\hline Intercept & $16.33 \pm 0.34$ & - & - & $19.86 \pm 0.19$ & - & - \\
\hline Treatment & $0.23 \pm 0.22$ & $1.1_{1,70}$ & 0.31 & $0.18 \pm 0.12$ & $2.3_{1,61}$ & 0.14 \\
\hline $\operatorname{Sex}$ & $0.15 \pm 0.11$ & $1.9_{1,196}$ & 0.17 & $0.34 \pm 0.09$ & $15_{1,168}$ & 0.0001 \\
\hline Hatching date & $0.01 \pm 0.02$ & $0.6_{1,69}$ & 0.44 & $-0.005 \pm 0.01$ & $0.2_{1,60}$ & 0.66 \\
\hline Brood size & $-0.12 \pm 0.07$ & $3.5_{1,70}$ & 0.067 & $-0.13 \pm 0.04$ & $12.5_{1,61}$ & 0.0008 \\
\hline Treatment $\times$ sex & $-0.03 \pm 0.23$ & $0.02_{1,95}$ & 0.9 & $-0.14 \pm 0.18$ & $0.6_{1,167}$ & 0.44 \\
\hline
\end{tabular}

$F$ - and $P$-values of non-significant terms are those just before removal from the model. Terms retained in the final model are highlighted in italic. Reference level for coefficients is a female under control treatment

a significantly higher chromatic reflectance than females $\left(F_{1,167}=4.9, P=0.03\right.$, estimate $\pm \mathrm{SE}=0.77 \pm 0.12$ for males, estimate $\pm \mathrm{SE}=0.74 \pm 0.12$ for females).

Fledging success and individual fledging probability

Broods hatched from lutein to supplemented eggs were significantly more likely to fledge $\left(\chi_{1}^{2}=3.96\right.$, $P=0.046$; Fig. 2). Fledging success showed a negative correlation with brood size $\left(\chi_{1}^{2}=4.7, P=0.03\right.$, estimate $\pm \mathrm{SE}=-0.30 \pm 0.14)$ but did not show any correlation with hatching date $\left(\chi_{1}^{2}=2.1, P=0.15\right)$. Individual fledging probability was predicted by treatment $\left(\chi_{1}^{2}=4.9, P=0.026\right.$, estimate $\pm \mathrm{SE}=2.21 \pm 0.99)$, and was not correlated with hatching date, brood size or sex (all $P>0.41$ ). To check if the effect of lutein treatment on fledging probability was driven by body mass, we ran an additional model of individual fledging probability including body mass at day 3 as a covariate. In this case fledging probability was predicted by body mass $\left(\chi_{1}^{2}=7.5, P=0.006\right.$, estimate $\left.\pm \mathrm{SE}=2.1 \pm 0.8\right)$ rather than treatment $\left(\chi_{1}^{2}=1.4, P=0.24\right)$.

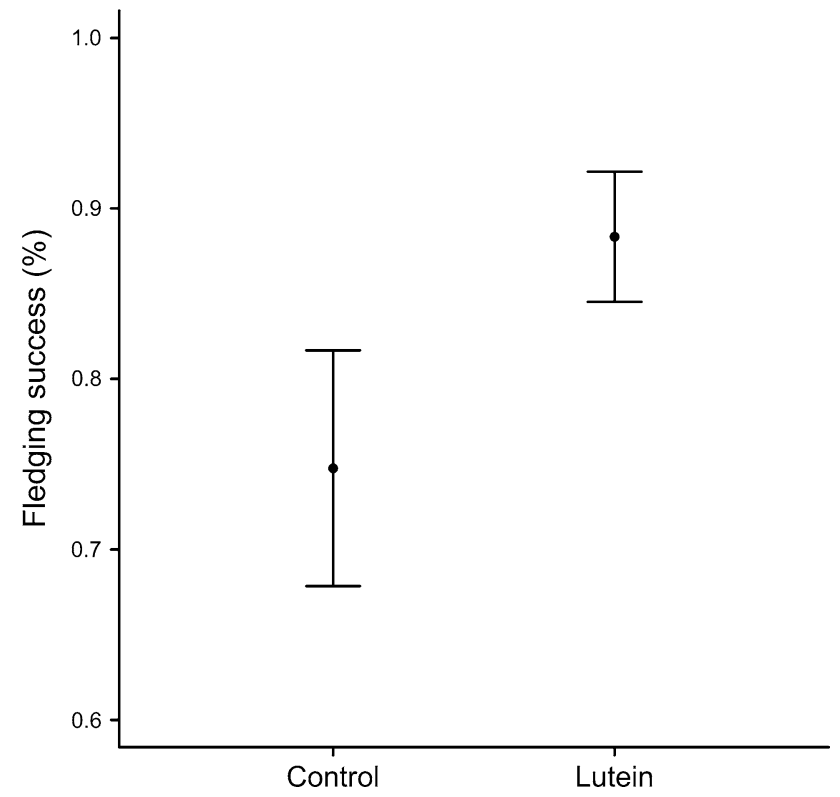

Fig. 2 Fledging success (mean $\pm \mathrm{SE}$ ), calculated as the proportion of hatchlings that fledged, in relation to the treatment 


\section{Discussion}

In this study we experimentally increased lutein content in great tit eggs. Lutein had a positive effect on body mass shortly after hatching, and increased fledging success. However, lutein did not affect tarsus length, oxidative stress, immune response or breast plumage colouration.

A higher content of lutein in the embryo tissues and in the newly hatched nestling may have increased growth by reducing oxidative stress. Rapidly growing embryos are particularly exposed to oxidative stress caused by high metabolic rates, and therefore need efficient antioxidants to protect developing tissues from oxidative damage (Surai 2002). The antioxidant activity of carotenoids in vivo has recently been questioned (Costantini and Møller 2008); however, it seems that they are mainly active at low oxygen tension, which prevails in embryonic tissues (Surai 2002). Lutein could have increased embryo or nestling growth also through its role in cell differentiation and proliferation. Studies where yolk lutein was experimentally increased are scarce and the effects are not consistent: in the barn swallow Hirundo rustica higher yolk lutein did not affect body mass (Saino et al. 2003), while in the yellow-legged gull Larus michahellis it increased growth of males hatched from first eggs, but decreased growth of males hatched from third eggs (Saino et al. 2011).

Oxidative stress was not affected by our treatment, supporting the idea that carotenoids are minor antioxidants in birds (Costantini and Møller 2008). Although, if yolk lutein was available to nestlings just for a short period after hatching, we would not expect any effect on oxidative stress, or immune response and colouration, since they were all measured 12 or more days after hatching. The absence of an effect of lutein on the immune response could also be explained by the fact that only $\beta$ carotene seems to have immuno-enhancing properties in great tit nestlings (Fitze et al. 2007). In the barn swallow lutein injection increased the immune response to phytohaemagglutinin (Saino et al. 2003), while in the great tit carotenoid supplementation to the mother did not affect offspring immune response (Remeš et al. 2007).

During embryonic life, carotenoids are transferred from the yolk to the developing tissues. Some of these carotenoids are either used in metabolic processes during embryo growth or stored in the liver, and redistributed after hatching. In poultry carotenoid concentration in the liver decreases 20 -fold during the first 10 days of life (reviewed in Surai and Fisinin 2013). Great tit nestlings have fast growth, which allows them to fledge 18-21 days post-hatch (Gosler 1993). We do not know for how long after hatching yolk carotenoids are available to nestlings. Our results can be explained if the concentration of yolk carotenoids available to nestlings rapidly decreases after hatching. Indeed, in our experiment, we found an effect on mass 3 days post-hatching, while all the other traits measured later during the nestling phase were not influenced by the treatment. The only exception is fledging success, which was positively influenced by lutein treatment, via a treatment effect on body mass. Indeed, individual fledging probability was correlated with body mass at day 3 and not with lutein treatment when both variables were included in the model. Possibly the first 3 days after hatching are a crucial period that can influence survival later in the nestling period. In both experimental groups, the majority of deaths occurred between day 8 and 14 post-hatch when the growth rate is high (Gosler 1993), indicating that there was no difference in the timing of mortality between control and lutein nestlings.

An alternative scenario is that yolk lutein is available to nestlings for a long period after hatching. Since lutein did not influence the traits measured 8 or more days post-hatch, this scenario seems unlikely. However, the reduced brood size caused by the injection could have masked the potential effects of lutein, as nestlings raised in small broods have more resources during growth. Probably the effects of supplementary yolk carotenoids are more easily detectable when resources are more limiting. Also, we cannot exclude that there could have been an effect of lutein on a trait we did not measure, for example resistance to parasites, which also may have influenced fledging success. Higher availability of carotenoids for females during laying increased the offspring survival in zebra finches Taeniopygia guttata (McGraw et al. 2005), but did not have an effect in great tits (Remeš 2011). However, direct manipulation of lutein content in eggs resulted in higher survival in female yellow-legged gull late in the season (Romano et al. 2008) and in a higher T cell-mediated immune response in the barn swallow, where immune response predicts offspring survival (Saino et al. 2003).

Our study shows the positive effect of yolk lutein on offspring survival in the great tit, suggesting an important role for maternal effects mediated by carotenoids. Further studies that directly manipulate carotenoid content of the eggs in different species are needed to better understand the role of yolk carotenoids.

Acknowledgments We would like to thank Laureline Durand and Javier Diaz Castillo for field assistance. Thanks also to Beatrice Voegeli and Sylvain Losdat for statistical help and to DSM Nutritional Products for generously providing carotenoids. This work was conducted under license of the Ethical Committee of the Agricultural Office of the Canton Bern (BE21/12). The work was funded by the Swiss National Science Foundation [grant 31003A_122566 to H. R.].

\section{References}

Berthouly A, Cassier A, Richner H (2008) Carotenoid-induced maternal effects interact with ectoparasite burden and brood size to shape the trade-off between growth and 
immunity in nestling great tits. Funct Ecol 22:854-863. doi:10.1111/J.1365-2435.2008.01439.X

Bertrand S, Alonso-Alvarez C, Devevey G, Faivre B, Prost J, Sorci G (2006) Carotenoids modulate the trade-off between egg production and resistance to oxidative stress in zebra finches. Oecologia 147:576-584. doi:10.1007/S00442-005-0317-8

Biard C, Surai PF, Møller AP (2005) Effects of carotenoid availability during laying on reproduction in the blue tit. Oecologia 144:3244. doi:10.1007/S00442-005-0048-X

Bonneaud C, Mazuc J, Gonzalez G, Haussy C, Chastel O, Faivre B, Sorci G (2003) Assessing the cost of mounting an immune response. Am Nat 161:367-379. doi:10.1086/346134

Costantini D (2006) Dell'Omo G (2006) Effects of T-cell-mediated immune response on avian oxidative stress. Comp Biochem Phys A 145:137-142. doi:10.1016/J.Cbpa.06.002

Costantini D, Møller AP (2008) Carotenoids are minor antioxidants for birds. Funct Ecol 22:367-370. doi:10.1111/ J.1365-2435.2007.01366.X

Evans SR, Hinks AE, Wilkin TA, Sheldon BC (2010) Age, sex and beauty: methodological dependence of age- and sex-dichromatism in the great tit Parus major. Biol J Linn Soc 101:777-796. doi:10.1111/J.1095-8312.2010.01548.X

Ewen JG, Thorogood R, Brekke P, Cassey P, Karadas F, Armstrong DP (2009) Maternally invested carotenoids compensate costly ectoparasitism in the hihi. Proc Natl Acad Sci USA 106:1279812802. doi:10.1073/Pnas.0902575106

Fitze PS, Tschirren B, Gasparini J, Richner H (2007) Carotenoidbased plumage colors and immune function: is there a trade-off for rare carotenoids? Am Nat 169:S137-S144

Goodwin TW (1984) The biochemistry of carotenoids. Chapman \& Hall, London

Gosler A (1993) The great tit. Hamlyn, London

Griffiths R, Double MC, Orr K, Dawson RJG (1998) A DNA test to sex most birds. Mol Ecol 7:1071-1075. doi:10.1046/ J.1365-294x.1998.00389.X

Groothuis TGG, Muller W, von Engelhardt N, Carere C, Eising C (2005) Maternal hormones as a tool to adjust offspring phenotype in avian species. Neurosci Biobehav R 29:329-352. doi:10.1016/J.Neubiorev.2004.12.002

Hafdfield JD (2005) The quantitative genetics of plumage colour in the blue tit (Parus major). PhD dissertation, Imperial College, London

Halliwell B, Gutteridge J (2007) Free radicals in biology and medicine. Oxford University Press, Oxford

Hart NS, Partridge JC, Cuthill IC, Bennett ATD (2000) Visual pigments, oil droplets, ocular media and cone photoreceptor distribution in two species of passerine bird: the blue tit (Parus caeruleus L.) and the blackbird (Turdus merula L.). J Comp Physiol A 186:375-387. doi:10.1007/S003590050437

Hasselquist D, Nilsson JA (2009) Maternal transfer of antibodies in vertebrates: trans-generational effects on offspring immunity. Philos T R Soc B 364:51-60. doi:10.1098/Rstb.2008.0137

Hill GE (1991) Plumage coloration is a sexually selected indicator of male quality. Nature 350:337-339. doi:10.1038/350337a0

Koutsos EA, Lopez JCG, Klasing KC (2006) Carotenoids from in ovo or dietary sources blunt systemic indices of the inflammatory response in growing chicks (Gallus gallus domesticus). J Nutr 136:1027-1031

Lessells CM, Boag PT (1987) Unrepeatable repeatabilities-a common mistake. Auk 104:116-121

McGraw KJ, Adkins-Regan E, Parker RS (2005) Maternally derived carotenoid pigments affect offspring survival, sex ratio, and sexual attractiveness in a colorful songbird. Naturwissenschaften 92:375-380. doi:10.1007/S00114-005-0003-Z

Møller AP, Biard C, Blount JD, Houston DC, Ninni P, Saino N, Surai PF (2000) Carotenoid-dependent signals: indicators of foraging efficiency, immunocompetence or detoxification ability? Avian Poult Biol Rev 11:137-159

Mousseau TA, Fox CW (1998) Maternal effects as adaptations. Oxford University Press, Oxford

Parmentier HK, Reilingh GD, Nieuwland MGB (1998) Kinetic and immunohistochemical characteristics of mitogen-induced cutaneous hypersensitivity in chickens selected for antibody responsiveness. Vet Immunol Immunopathol 66:367-376. doi:10.1016/ S0165-2427(98)00200-1

Podlas K, Helfenstein F, Richner H (2013) Brood reduction via intraclutch variation in testosterone - an experimental test in the great tit. Plos One 8(2):e56672. doi:10.1371/journal.pone.0056672

Remeš V (2011) Yolk androgens in great tit eggs are related to male attractiveness, breeding density and territory quality. Behav Ecol Sociobiol 65:1257-1266. doi:10.1007/S00265-011-1139-9

Remeš V, Krist M, Bertacche V, Stradi R (2007) Maternal carotenoid supplementation does not affect breeding performance in the Great Tit (Parus major). Funct Ecol 21:776-783. doi:10.1111/J.1365-2435.2007.01277.X

Remeš V, Matysiokova B, Klejdus B (2011) Egg yolk antioxidant deposition as a function of parental ornamentation, age, and environment in great tits Parus major. J Avian Biol 42:387-396. doi:10.1111/J.1600-048x.2011.05402.X

Romano M, Caprioli M, Ambrosini R, Rubolini D, Fasola M, Saino N (2008) Maternal allocation strategies and differential effects of yolk carotenoids on the phenotype and viability of yellow-legged gull (Larus michahellis) chicks in relation to sex and laying order. J Evol Biol 21:1626-1640. doi:10.1111/ J.1420-9101.2008.01599.X

Ruuskanen S, Doligez B, Pitala N, Gustafsson L, Laaksonen T (2012) Long-term fitness consequences of high yolk androgen levels: sons pay the costs. Funct Ecol 26:884-894. doi:10.1111/J.1365-2435.2012.01994.X

Saino N, Ninni P, Calza S, Martinelli R, De Bernardi F, Møller AP (2000) Better red than dead: carotenoid-based mouth coloration reveals infection in barn swallow nestlings. Proc R Soc B 267:57-61

Saino N, Bertacche V, Ferrari RP, Martinelli R, Møller AP, Stradi R (2002) Carotenoid concentration in barn swallow eggs is influenced by laying order, maternal infection and paternal ornamentation. Proc R Soc B 269:1729-1733. doi:10.1098/Rspb.2002.2088

Saino N, Ferrari R, Romano M, Martinelli R, Møller AP (2003) Experimental manipulation of egg carotenoids affects immunity of barn swallow nestlings. Proc R Soc Lond B 270:2485-2489. doi:10.1098/Rspb.2003.2534

Saino N, Romano M, Caprioli M, Rubolini D, Ambrosini R (2011) Yolk carotenoids have sex-dependent effects on redox status and influence the resolution of growth trade-offs in yellow-legged gull chicks. Behav Ecol 22:411-421. doi:10.1093/Beheco/Arq220

Saks L, McGraw K, Hõrak P (2003) How feather colour reflects its carotenoid content. Funct Ecol 17:555-561. doi:10.1046/ J.1365-2435.2003.00765.X

Sies H (1991) Oxidative stress-from basic research to clinical-application. Am J Med 91:S31-S38. doi:10.1016/0002-9343(91)90281-2

Surai PF (2002) Natural antioxidants in avian nutrition and reproduction. Nottingham University Press, Nottingham

Surai PF, Fisinin VI (2013) Natural antioxidants in hens' embryogenesis and antistress defence in postnatal development. Sel'skokhozyaistven Biol 2:3-18

Surai AP, Surai PF, Steinberg W, Wakeman WG, Speake BK, Sparks NHC (2003) Effect of canthaxanthin content of the maternal diet on the antioxidant system of the developing chick. Br Poultry Sci 44:612-619. doi:10.1080/00071660310001616200 